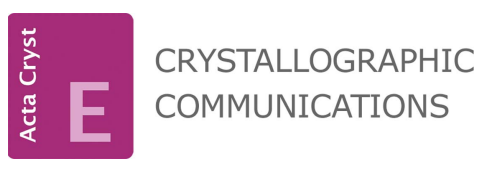

ISSN 2056-9890

Received 20 March 2019

Accepted 7 May 2019

Edited by M. Weil, Vienna University of Technology, Austria

Keywords: crystal structure; phthalonitrile; imidazole; Hirshfeld analysis; hydrogen bonds.

CCDC reference: 1846754

Supporting information: this article has supporting information at journals.iucr.org/e

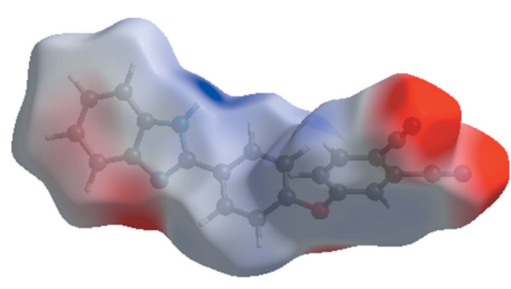

OPEN $\odot$ ACCESS

\section{Crystal structure and Hirshfeld surface analysis of 4-[4-(1H-benzo[d] imidazol-2-yl)phenoxy]phthalo- nitrile dimethyl sulfoxide monosolvate}

\author{
Sibel Demir Kanmazalp, ${ }^{\text {a* }}$ Pınar Sen, ${ }^{\mathrm{b}}$ Necmi Dege, ${ }^{\mathrm{c} *}$ Salih Zeki Yildiz, ${ }^{\mathrm{d}}$ Namık \\ Ozdemir $^{\mathrm{e}}$ and Irina A. Golenya ${ }^{\mathrm{f}_{*}}$
}

aGaziantep University, Technical Sciences, 27310, Gaziantep, Turkey, 'bentre for Nanotechnology Innovation, Department of Chemistry, Rhodes University, Grahamstown, South Africa, ' Ondokuz Mayıs University, Faculty of Arts and Sciences, Department of Physics, 55139 Samsun, Turkey, ${ }^{\mathbf{d}}$ Sakarya University, Faculty of Arts and Sciences, Department of Chemistry, 54187, Sakarya, Turkey, ${ }^{\mathbf{e} D e p a r t m e n t ~ o f ~ M a t h e m a t i c s ~ a n d ~ S c i e n c e ~ E d u c a t i o n, ~ F a c u l t y ~ o f ~}$ Education, Ondokuz Mayıs University, Samsun, Turkey, and 'Taras Shevchenko National University of Kyiv, Department of Chemistry, 64, Vladimirska Str., Kiev 01601, Ukraine. *Correspondence e-mail: sibeld@gantep.edu.tr, necmid@omu.edu.tr, igolenya@ua.fm

This work presents the synthesis and structural characterization of [4-(1Hbenzo[d]imidazol-2-yl)phenoxy]phthalonitrile, a phthalonitrile derivative carrying a benzimidazole moiety. The compound crystallizes as its dimethyl sulfoxide monosolvate, $\mathrm{C}_{21} \mathrm{H}_{12} \mathrm{~N}_{4} \mathrm{O} \cdot\left(\mathrm{CH}_{3}\right)_{2} \mathrm{SO}$. The dihedral angle between the two fused rings in the heterocyclic ring system is $2.11(1)^{\circ}$, while the phenyl ring attached to the imidazole moiety is inclined by $20.7(1)^{\circ}$ to the latter. In the crystal structure, adjacent molecules are connected by pairs of weak intermolecular $\mathrm{C}-\mathrm{H} \cdots \mathrm{N}$ hydrogen bonds into inversion dimers. $\mathrm{N}-\mathrm{H} \cdots \mathrm{O}$ and $\mathrm{C}-$ $\mathrm{H}$. . O hydrogen bonds with $R_{2}^{1}(7)$ graph-set motifs are also formed between the organic molecule and the disordered dimethyl sulfoxide solvent [occupancy ratio of 0.623 (5):0.377 (5) for the two sites of the sulfur atom]. Hirshfeld surface analysis and fingerprint plots were used to investigate the intermolecular interactions in the crystalline state.

\section{Chemical context}

Benzimidazole and its derivatives are some of the oldest and chemically most-studied nitrogen-containing aromatic heterocyclic compounds (Srestha et al., 2014). They have a wide range of applications in medicinal chemistry and in biological processes including as anticancer, antiulcer, antifungal and anti-inflammatory agents, and exhibit antimycobacterial and antioxidant activities (El Rashedy \& Aboul-Enein, 2013; Gaba et al., 2014; Kathiravan et al., 2012). They are also used as ligands with fluorescent properties. The fluorescent characteristic of these compounds can be changed by substitution or derivatization of different groups at the $\mathrm{NH}$ position of the benzimidazole skeleton.

Phthalonitrile derivatives are some of the most widely used precursors for the preparation of phthalocyanines (Pc). The preparation of phthalocyanines is frequently carried out by a cyclotetramerization reaction of phthalonitriles. The synthesis of the latter compound family, carrying different functional groups, leads to functionalized phthalocyanines that are of great importance with respect to new molecular materials and targeted applications such as catalysis, liquid crystals, photosensitizers for photodynamic therapy (PDT), non-linear optics, nanotechnology or dye-sensitized solar cells (Torre et 
al., 2004; Martínez-Díaz et al., 2011). In this context, we have recently described a model study, i.e. the synthesis, characterization and Hirshfeld surface analysis of zinc phthalocyanines carrying benzimidazole groups through oxygen bridges to a $\mathrm{Zn}-\mathrm{Pc}$ core (Sen et al., 2018b). Here we report the synthesis, structural characterization and Hirshfeld surface analysis of a related ligand that crystallizes as its dimethylsulfoxide monosolvate, $\mathrm{C}_{21} \mathrm{H}_{12} \mathrm{~N}_{4} \mathrm{O} \cdot\left(\mathrm{CH}_{3}\right)_{2} \mathrm{SO}$.

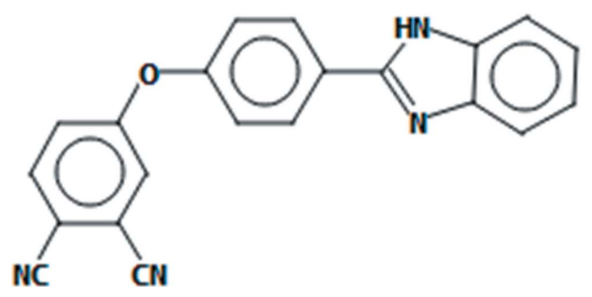

\section{Structural commentary}

The molecular components of the title compound are shown in Fig. 1. The molecular structure of the phthalonitrile derivative is constructed from three ring systems, viz. a central phenoxy ring, a terminal phthalonitrile system and a terminal benzimidazole ring. The bond lengths of the cyano groups, 1.132 (6) and 1.137 (6) $\AA$, for $\mathrm{C} 21 \equiv \mathrm{N} 4$ and $\mathrm{C} 20 \equiv \mathrm{N} 3$, respectively, conform well with literature values (Saraçoğlu et al., 2011). The corresponding $\mathrm{C}-\mathrm{C} \equiv \mathrm{N}$ angles [179.4 (6) and $177.9(7)^{\circ}$ ] are almost linear and are also in good agreement with literature values (Saraçoğlu et al., 2011; Sen et al., 2018a). The C-C bond lengths of the phenyl rings are in the normal range of 1.356 (5)-1.395 (6) A, i.e. characteristic of a delocalized system. The dihedral angle of $2.11(1)^{\circ}$ between the fused $\mathrm{C} 1-$ $\mathrm{C} 6$ and $\mathrm{C} 5 / \mathrm{N} 2 / \mathrm{C} 7 / \mathrm{N} 1 / \mathrm{C} 6$ rings in the heterocycle indicate a minute deviation from planarity, whereas the attached $\mathrm{C} 8-\mathrm{C} 13$ ring is inclined by $20.7(1)^{\circ}$ to the $\mathrm{C} 5 / \mathrm{N} 2 / \mathrm{C} 7 / \mathrm{N} 1 / \mathrm{C} 6$ ring plane.

\section{Supramolecular features}

In the crystal structure, $\mathrm{N} 2-\mathrm{H} 2 \cdots \mathrm{O} 2$ and $\mathrm{C} 9-\mathrm{H} 9 \cdots \mathrm{O} 2$ intermolecular hydrogen bonding interactions with an $R_{2}^{1}(7)$

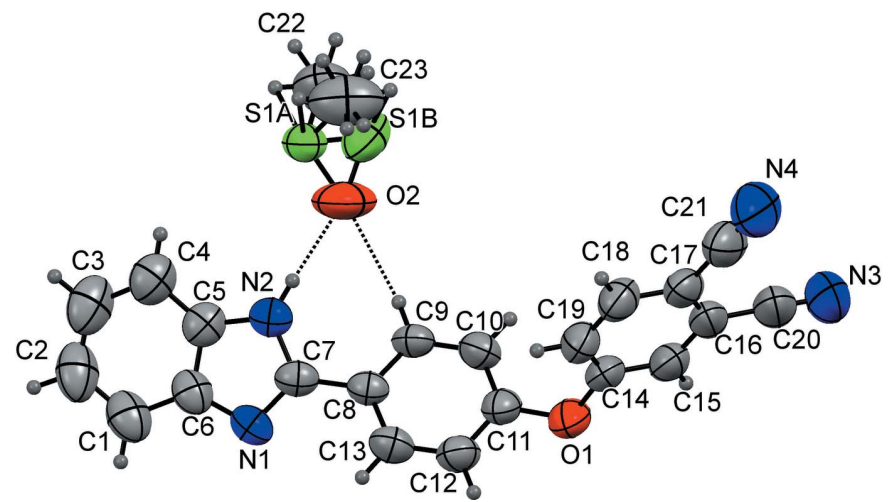

Figure 1

The molecular structure of the title compound, showing the atom labelling. Displacement ellipsoids are drawn at the $50 \%$ probability level. Hydrogen bonds (Table 1) are shown as dashed lines.

Table 1

Hydrogen-bond geometry $\left(\AA,^{\circ}\right)$.

\begin{tabular}{lllll}
\hline$D-\mathrm{H} \cdots A$ & $D-\mathrm{H}$ & $\mathrm{H} \cdots A$ & $D \cdots A$ & $D-\mathrm{H} \cdots A$ \\
\hline $\mathrm{N} 2-\mathrm{H} 2 \cdots \mathrm{O} 2$ & 0.86 & 1.94 & $2.794(5)$ & 172 \\
$\mathrm{~N} 2-\mathrm{H} 2 \cdots \mathrm{S} 1 A$ & 0.86 & 2.83 & $3.614(4)$ & 152 \\
$\mathrm{C} 9-\mathrm{H} 9 \cdots \mathrm{O} 2$ & 0.93 & 2.40 & $3.175(5)$ & 141 \\
$\mathrm{C} 23-\mathrm{H} 23 D \cdots \mathrm{N} 4$ & 0.96 & 2.63 & $3.500(9)$ & 151 \\
\hline
\end{tabular}

Symmetry code: (i) $-x+\frac{1}{2}, y-\frac{1}{2}, z+\frac{1}{2}$.

graph-set motif are present, whereby the $\mathrm{O} 2$ atom acts as an acceptor in both cases (Fig. 1). There are also weak intermolecular $\mathrm{N} 2-\mathrm{H} 2 \cdots \mathrm{S} 1 A$ interactions between the the $\mathrm{N}-\mathrm{H}$ group of the imidazole ring and the disordered dimethyl sulfate solvent, and a $\mathrm{C} 23-\mathrm{H} 23 D \cdots \mathrm{N} 4$ interaction between one of the methyl groups of the dimethyl sulfoxide solvent and

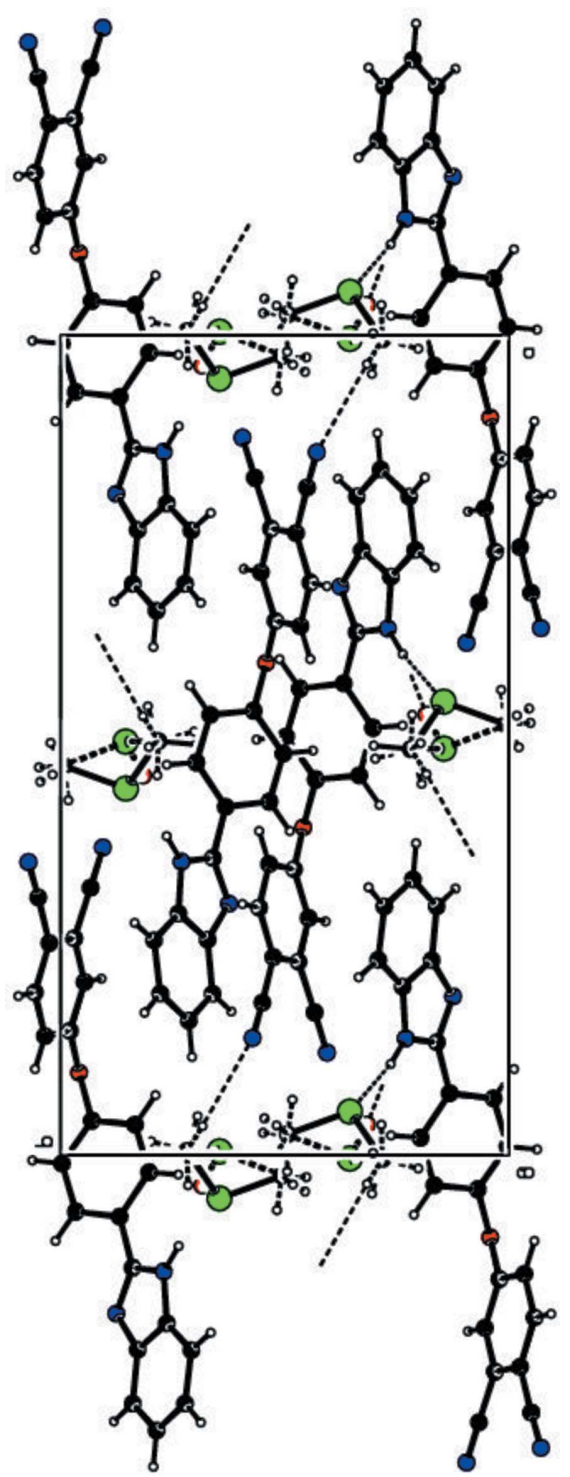

Figure 2

A view of the crystal packing of the title compound. Dashed lines denote the $\mathrm{N} 2-\mathrm{H} 2 \cdots \mathrm{S} 1 A, \mathrm{~N} 2-\mathrm{H} 2 \cdots \mathrm{O} 2$ and $\mathrm{C} 23-\mathrm{H} 23 D \cdots \mathrm{N} 4$ intermolecular hydrogen-bonding interactions. 


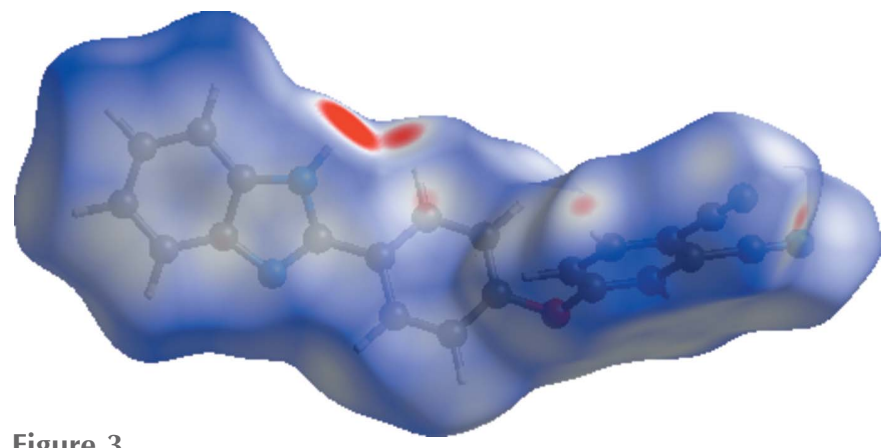

Figure 3

The Hirshfeld surface of the title compound mapped with $d_{\text {norm }}$ in the range -0.6328 to 1.3784 a.u.

one of the nitrile $\mathrm{N}$ atoms (Table 1, Fig. 2). These interactions lead to the formation of a three-dimensional supramolecular network.

\section{Database survey}

A search of the Cambridge Structural database (CSD, version 5.40, update November 2018; Groom et al., 2016) for the 4-[4(1H-benzo $[d]$ imidazole-2yl)phenoxy]phthalonitrile moiety revealed two hits. Distinctive bond lengths ( $\mathrm{N} 4 \equiv \mathrm{C} 21$, $\mathrm{N} 3 \equiv \mathrm{C} 20, \mathrm{C} 7-\mathrm{N} 2, \mathrm{C} 5-\mathrm{N} 2)$ in the title structure are the same within standard uncertainties as the corresponding bond lengths in the structures of 4-[4-(1H-benzimidazol-2-yl)phenoxy]benzene-1,2-dicarbonitrile monohydrate (HIDHEK; Sen et al., 2018b) or 4-\{4-[1-(prop-2-en-1-yl)-1H-benzimidazol-2yl]phenoxy\}benzene-1,2-dicarbonitrile (RELBUI; Sen et al., $2018 a$ ). In these structures, the $\mathrm{C}-\mathrm{O}$ bond lengths vary from
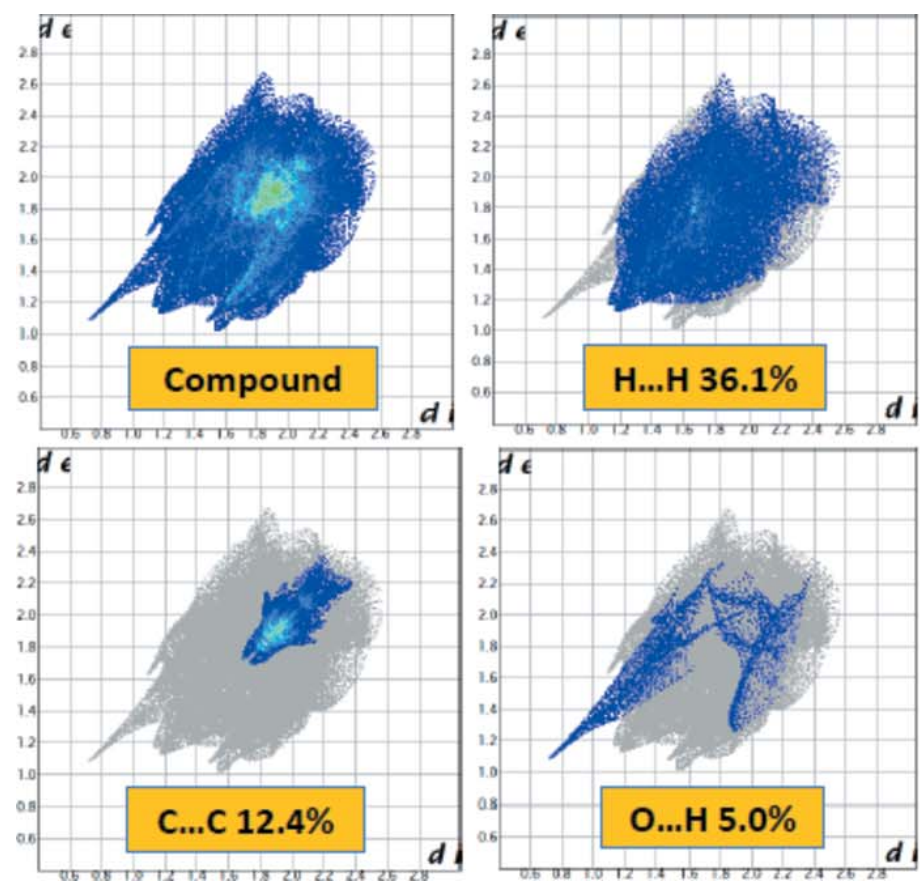

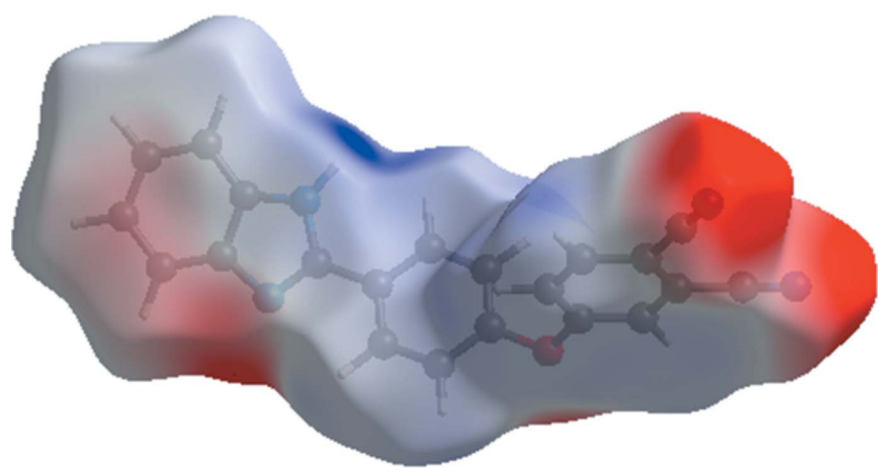

Figure 5

A view of the three-dimensional Hirshfeld surface of the title compound plotted over electrostatic potentials in the range -0.0893 to 0.1930 a.u.

1.363-1.407 $\AA$. In the title molecule, the corresponding bond lengths are 1.367 (5) and 1.406 (4) $\AA$, respectively. In all these structures, the molecules are linked into chains by $\mathrm{C}-\mathrm{H} \cdots \mathrm{N}$ hydrogen bonds.

\section{Hirshfeld surface analysis}

The Hirshfeld surface analysis (Spackman \& Jayatilaka, 2009) and the associated two-dimensional fingerprint plots (McKinnon et al., 2007) were performed with CrystalExplorer17 (Turner et al., 2017). The Hirshfeld surfaces were generated using a standard (high) surface resolution with the three-dimensional surfaces mapped over $d_{\text {norm }}$ (Fig. 3). For the title molecule, the $\mathrm{H} \cdots \mathrm{H}$ interactions appear in the middle of the scattered points in the fingerprint plots with a contribution to the overall Hirshfeld surface of $36.1 \%$ (Fig. 4). The
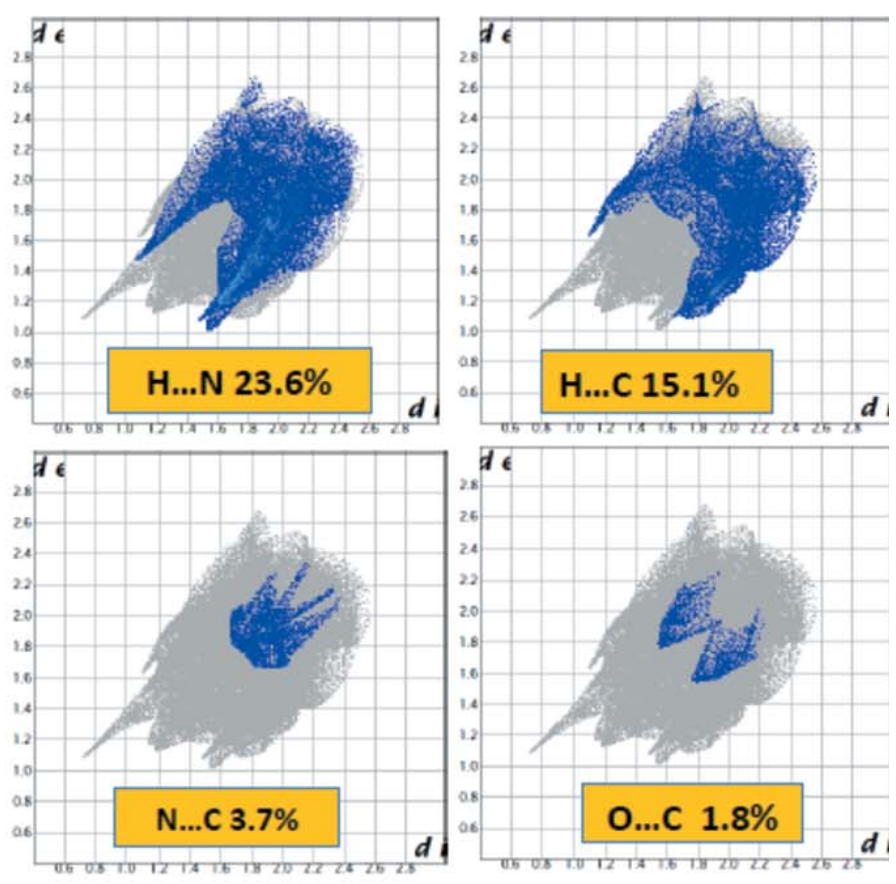

Figure 4

Two-dimensional fingerprint plots with a $d_{\text {norm }}$ view of all interactions in the title compound, and subdivided into $\mathrm{H} \cdots \mathrm{H}(36.1 \%), \mathrm{N} \cdots \mathrm{H} / \mathrm{H} \cdots \mathrm{N}(23.6 \%)$, $\mathrm{C} \cdots \mathrm{H} / \mathrm{H} \cdots \mathrm{C}(15.1 \%), \mathrm{C} \cdots \mathrm{C} / \mathrm{C} \cdots \mathrm{C}(12.4 \%), \mathrm{O} \cdots \mathrm{H} / \mathrm{H} \cdots \mathrm{O}(5.0 \%), \mathrm{C} \cdots \mathrm{N} / \mathrm{N} \cdots \mathrm{C}(3.7 \%), \mathrm{C} \cdots \mathrm{O} / \mathrm{O} \cdots \mathrm{C}(1.8 \%)$ and $\mathrm{S} \cdots \mathrm{H} / \mathrm{H} \cdots \mathrm{S}(1.6 \%)$ contacts. 
Table 2

Experimental details.

\begin{tabular}{|c|c|}
\hline \multicolumn{2}{|l|}{ Crystal data } \\
\hline Chemical formula & $\mathrm{C}_{21} \mathrm{H}_{12} \mathrm{~N}_{4} \mathrm{O} \cdot \mathrm{C}_{2} \mathrm{H}_{6} \mathrm{OS}$ \\
\hline$M_{\mathrm{r}}$ & 414.47 \\
\hline Crystal system, space group & Orthorhombic, Pna2 $2_{1}$ \\
\hline Temperature (K) & 296 \\
\hline$a, b, c(\AA)$ & $\begin{array}{l}20.9154(11), 11.4208(6) \\
\quad 8.8938(6)\end{array}$ \\
\hline$V\left(\AA^{3}\right)$ & $2124.5(2)$ \\
\hline$Z$ & 4 \\
\hline Radiation type & Мо $K \alpha$ \\
\hline$\mu\left(\mathrm{mm}^{-1}\right)$ & 0.18 \\
\hline Crystal size (mm) & $0.65 \times 0.56 \times 0.47$ \\
\hline \multicolumn{2}{|l|}{ Data collection } \\
\hline Diffractometer & Stoe IPDS 2 \\
\hline Absorption correction & $\begin{array}{l}\text { Integration ( } X \text {-RED32; Stoe \& Cie, } \\
\text { 2002) }\end{array}$ \\
\hline$T_{\min }, T_{\max }$ & $0.966,0.977$ \\
\hline $\begin{array}{l}\text { No. of measured, independent and } \\
\text { observed }[I>2 \sigma(I)] \text { reflections }\end{array}$ & $15225,4660,2281$ \\
\hline$R_{\text {int }}$ & 0.058 \\
\hline$(\sin \theta / \lambda)_{\max }\left(\AA^{-1}\right)$ & 0.641 \\
\hline \multicolumn{2}{|l|}{ Refinement } \\
\hline$R\left[F^{2}>2 \sigma\left(F^{2}\right)\right], w R\left(F^{2}\right), S$ & $0.042,0.098,0.83$ \\
\hline No. of reflections & 4660 \\
\hline No. of parameters & 281 \\
\hline No. of restraints & 1 \\
\hline $\mathrm{H}$-atom treatment & $\mathrm{H}$-atom parameters constrained \\
\hline$\Delta \rho_{\max }, \Delta \rho_{\min }\left(\mathrm{e} \AA^{-3}\right)$ & $0.20,-0.12$ \\
\hline Absolute structure & $\begin{array}{l}\text { Flack } x \text { determined using } 771 \\
\text { quotients }\left[\left(I^{+}\right)-\left(I^{-}\right)\right] /\left[\left(I^{+}\right)+\left(I^{-}\right)\right] \\
\text {(Parsons } \text { et al., 2013) }\end{array}$ \\
\hline Absolute structure parameter & $-0.02(8)$ \\
\hline
\end{tabular}

Computer programs: X-AREA and X-RED32 (Stoe \& Cie, 2002), SHELXT2018 (Sheldrick, 2015a), SHELXL2018 (Sheldrick, 2015b), ORTEP-3 for Windows and WinGX (Farrugia, 2012), Mercury (Macrae et al., 2006) and PLATON (Spek, 2009).

contribution from the $\mathrm{N} \cdots \mathrm{H} / \mathrm{H} \cdots \mathrm{N}$ contacts, corresponding to the $\mathrm{C}-\mathrm{H} \cdots \mathrm{N}$ interactions, is represented by a pair of sharp spikes characteristic of a rather strong hydrogen-bonding interaction $(23.6 \%)$. The whole fingerprint region and all other interactions are displayed in Fig. 4. In particular, the $\mathrm{O} \cdots \mathrm{H} / \mathrm{H} \cdots \mathrm{O}$ contacts indicate the presence of intermolecular $\mathrm{C}-\mathrm{H} \cdots \mathrm{O}$ and $\mathrm{N}-\mathrm{H} \cdots \mathrm{O}$ interactions.

A view of the molecular electrostatic potential for the title compound, using the STO-3G basis set at the Hartree-Fock level of theory, is shown in Fig. 5. The $\mathrm{N}-\mathrm{H} \cdots \mathrm{N}$ and $\mathrm{C}-$ $\mathrm{H} \cdots \mathrm{N}$ hydrogen-bond donor and acceptor groups are shown as blue and red areas around the atoms related with positive (hydrogen-bond donors) and negative (hydrogen-bond acceptors) electrostatic potentials, respectively.

\section{Synthesis and crystallization}

2-(4-Hydroxy-phenyl)-benzimidazole $\quad(1.2 \mathrm{~g}, \quad 5.71 \mathrm{mmol})$, which was synthesized by the reaction of $o$-phenylenediamine and 4-hydroxybenzaldehyde, and 4-nitrophthalonitrile $(0.989 \mathrm{~g}, 5.71 \mathrm{mmol})$ were dissolved in DMF $(15 \mathrm{ml})$ and degassed by argon in a dual-bank vacuum-gas manifold system. After stirring for $15 \mathrm{~min}$, finely ground anhydrous $\mathrm{K}_{2} \mathrm{CO}_{3}(0.790 \mathrm{~g}, 5.71 \mathrm{mmol})$ was added portion-wise over $2 \mathrm{~h}$ under stirring. The suspension solution was maintained at $333 \mathrm{~K}$ for $24 \mathrm{~h}$. After completion of the reaction, the crude product was precipitated by pouring into ice-water. The precipitate was collected by filtration, washed with hot water, ethanol, diethyl ether and was finally dried in vacuo. The desired compound was obtained in sufficient purity. The obtained spectroscopic data are accordance with the literature (Khan et al., 2009). Single crystals for structure analysis were obtained from slow evaporation of a DMSO solution.

\section{Refinement}

Crystal data, data collection and structure refinement details are summarized in Table 2. $\mathrm{H}$ atoms were positioned geometrically and allowed to ride on their parent atoms, with $\mathrm{C}-\mathrm{H}=$ $0.93 \AA$ for aromatic groups, with $\mathrm{N}-\mathrm{H}=0.86 \AA$ for the imidazole moiety and with $0.96 \AA$ for methyl groups. $U_{\text {iso }}(\mathrm{H})$ values were constrained to $1.2-1.5 \mathrm{U}_{\mathrm{eq}}$ of their carrier atoms. The sulfur atom of the dimethylsulfate solvent is disordered over two sites (S1A and $\mathrm{S} 1 B)$, with an occupancy ratio of 0.623 (5):0.377 (5).

\section{Acknowledgements}

The authors acknowledge the Faculty of Arts and Sciences, Ondokuz Mayis University, Turkey, for the use of the Stoe IPDS 2 diffractometer (purchased under grant F.279 of the University Research Fund).

\section{Funding information}

This study was supported by Ondokuz Mayis University under project No. PYOFEN.1906.19.001 (contract No. PYOFEN.1906.19.001).

\section{References}

El Rashedy, A. A. \& Aboul-Enein, H. Y. (2013). Mini Rev. Med. Chem. 13, 399-407.

Farrugia, L. J. (2012). J. Appl. Cryst. 45, 849-854.

Gaba, M., Singh, S. \& Mohan, C. (2014). Eur. J. Med. Chem. 76, 494 505.

Groom, C. R., Bruno, I. J., Lightfoot, M. P. \& Ward, S. C. (2016). Acta Cryst. B72, 171-179.

Kathiravan, M. K., Salake, A. B., Chothe, A. S., Dudhe, P. B., Watode, R. P., Mukta, M. S. \& Gadhwe, S. (2012). Bioorg. Med. Chem. 20, 5678-5698.

Khan, A. T., Parvin, T. \& Choudhury, L. H. (2009). Synth. Commun. 39, 2339-2346.

Macrae, C. F., Edgington, P. R., McCabe, P., Pidcock, E., Shields, G. P., Taylor, R., Towler, M. \& van de Streek, J. (2006). J. Appl. Cryst. 39, 453-457.

Martínez-Díaz, M. V., Ince, M. \& Torres, T. (2011). Monatsh. Chem. 142, 699-707.

McKinnon, J. J., Jayatilaka, D. \& Spackman, M. A. (2007). Chem. Commun., pp. 3814-3816.

Parsons, S., Flack, H. D. \& Wagner, T. (2013). Acta Cryst. B69, 249 259.

Saraçoğlu, H., Güntepe, F., Yüksektepe, Ç. N. \& Saydam, S. (2011). Mol. Cryst. Liq. Cryst. 537, 111-127.

Sen, P., Atmaca, G. Y., Erdogmus, A., Kanmazalp, S. D., Dege, N. \& Yildiz, S. Z. (2018b). J. Lumin. 194, 123-130. 
Sen, P., Kansiz, S., Dege, N., Iskenderov, T. S. \& Yildiz, S. Z. (2018a). Acta Cryst. E74, 994-997.

Sheldrick, G. M. (2015a). Acta Cryst. A71, 3-8.

Sheldrick, G. M. (2015b). Acta Cryst. C71, 3-8.

Spackman, M. A. \& Jayatilaka, D. (2009). CrystEngComm, 11, 19-32.

Spek, A. L. (2009). Acta Cryst. D65, 148-155.

Srestha, N., Banerjee, J. \& Srivastava, S. (2014). IOSR J. Pharma 4, $28-41$.
Stoe \& Cie (2002). $X$-AREA and $X$-RED32. Stoe \& Cie GmbH, Darmstadt, Germany.

Torre, G. de la, Vázquez, P., Agulló-López, F. \& Torres, T. (2004). Chem. Rev. 104, 3723-3750.

Turner, M. J., McKinnon, J. J., Wolff, S. K., Grimwood, D. J., Spackman, P. R., Jayatilaka, D. \& Spackman, M. A. (2017). CrystalExplorer17. University of Western Australia. http://hirshfeldsurface.net 


\section{supporting information}

Acta Cryst. (2019). E75, 780-784 [https://doi.org/10.1107/S2056989019006510]

Crystal structure and Hirshfeld surface analysis of 4-[4-(1H-benzo[d]imidazol-2yl)phenoxy]phthalonitrile dimethyl sulfoxide monosolvate

Sibel Demir Kanmazalp, Pınar Şen, Necmi Dege, Salih Zeki Yildiz, Namık Ozdemir and Irina A. Golenya

Computing details

Data collection: $X$-AREA (Stoe \& Cie, 2002); cell refinement: $X$-AREA (Stoe \& Cie, 2002); data reduction: $X$-RED32 (Stoe \& Cie, 2002); program(s) used to solve structure: SHELXT2018 (Sheldrick, 2015a); program(s) used to refine structure: SHELXL2018 (Sheldrick, 2015b); molecular graphics: ORTEP-3 for Windows (Farrugia, 2012) and Mercury (Macrae et al., 2006); software used to prepare material for publication: SHELXL2018 (Sheldrick, 2015b), WinGX (Farrugia, 2012) and PLATON (Spek, 2009).

4-[4-(1H-Benzo[d]imidazol-2-yl)phenoxy]phthalonitrile dimethyl sulfoxide monosolvate

Crystal data

$\mathrm{C}_{21} \mathrm{H}_{12} \mathrm{~N}_{4} \mathrm{O} \cdot \mathrm{C}_{2} \mathrm{H}_{6} \mathrm{OS}$

$M_{r}=414.47$

Orthorhombic, Pna2 1

$a=20.9154(11) \AA$

$b=11.4208(6) \AA$

$c=8.8938(6) \AA$

$V=2124.5(2) \AA^{3}$

$Z=4$

$F(000)=864$

Data collection

Stoe IPDS 2 diffractometer

Radiation source: sealed X-ray tube, 12 x 0.4 $\mathrm{mm}$ long-fine focus

Plane graphite monochromator

Detector resolution: 6.67 pixels $\mathrm{mm}^{-1}$

rotation method scans

Absorption correction: integration

(X-RED32; Stoe \& Cie, 2002)

Refinement

Refinement on $F^{2}$

Least-squares matrix: full

$R\left[F^{2}>2 \sigma\left(F^{2}\right)\right]=0.042$

$w R\left(F^{2}\right)=0.098$

$S=0.83$

4660 reflections
$D_{\mathrm{x}}=1.296 \mathrm{Mg} \mathrm{m}^{-3}$

Mo $K \alpha$ radiation, $\lambda=0.71073 \AA$

Cell parameters from 9474 reflections

$\theta=1.8-27.0^{\circ}$

$\mu=0.18 \mathrm{~mm}^{-1}$

$T=296 \mathrm{~K}$

Prism, yellow

$0.65 \times 0.56 \times 0.47 \mathrm{~mm}$

$T_{\min }=0.966, T_{\max }=0.977$

15225 measured reflections

4660 independent reflections

2281 reflections with $I>2 \sigma(I)$

$R_{\text {int }}=0.058$

$\theta_{\text {max }}=27.1^{\circ}, \theta_{\min }=2.0^{\circ}$

$h=-26 \rightarrow 22$

$k=-14 \rightarrow 14$

$l=-11 \rightarrow 11$

281 parameters

1 restraint

Hydrogen site location: inferred from neighbouring sites

$\mathrm{H}$-atom parameters constrained 
$w=1 /\left[\sigma^{2}\left(F_{\mathrm{o}}^{2}\right)+(0.0409 P)^{2}\right]$

where $P=\left(F_{\mathrm{o}}^{2}+2 F_{\mathrm{c}}^{2}\right) / 3$

$(\Delta / \sigma)_{\max }<0.001$

$\Delta \rho_{\max }=0.20 \mathrm{e} \AA^{-3}$

$\Delta \rho_{\min }=-0.12$ e $\AA^{-3}$
Absolute structure: Flack $x$ determined using 771 quotients $\left[\left(I^{+}\right)-(I)\right] /\left[\left(I^{+}\right)+(I)\right]$ (Parsons et al., 2013)

Absolute structure parameter: $-0.02(8)$

Special details

Geometry. All esds (except the esd in the dihedral angle between two 1.s. planes) are estimated using the full covariance matrix. The cell esds are taken into account individually in the estimation of esds in distances, angles and torsion angles; correlations between esds in cell parameters are only used when they are defined by crystal symmetry. An approximate (isotropic) treatment of cell esds is used for estimating esds involving l.s. planes.

Fractional atomic coordinates and isotropic or equivalent isotropic displacement parameters $\left(\AA^{2}\right)$

\begin{tabular}{|c|c|c|c|c|c|}
\hline & $x$ & $y$ & $z$ & $U_{\text {iso }} * / U_{\text {eq }}$ & Occ. $(<1)$ \\
\hline S1A & $0.55296(11)$ & $0.14556(16)$ & $0.7814(2)$ & $0.0869(9)$ & $0.623(5)$ \\
\hline S1B & $0.4957(2)$ & $0.1452(4)$ & $0.7420(4)$ & $0.114(2)$ & $0.377(5)$ \\
\hline $\mathrm{O} 1$ & $0.39958(15)$ & $0.4586(3)$ & $0.0943(3)$ & $0.0925(8)$ & \\
\hline $\mathrm{O} 2$ & $0.53727(19)$ & $0.1838(3)$ & $0.6323(4)$ & $0.1425(15)$ & \\
\hline N1 & $0.69349(16)$ & $0.3756(2)$ & 0.2997 (4) & $0.0751(8)$ & \\
\hline N2 & $0.64212(17)$ & $0.2699(2)$ & $0.4721(3)$ & $0.0720(8)$ & \\
\hline $\mathrm{H} 2$ & 0.611036 & 0.236598 & 0.518771 & $0.086^{*}$ & \\
\hline N3 & $0.1249(2)$ & $0.4056(4)$ & $0.1258(7)$ & 0.1429 (19) & \\
\hline N4 & $0.1428(2)$ & $0.5716(5)$ & $0.5187(7)$ & $0.156(2)$ & \\
\hline $\mathrm{C} 1$ & $0.8034(2)$ & $0.3391(4)$ & $0.4030(6)$ & $0.0968(13)$ & \\
\hline H1 & 0.824993 & 0.380986 & 0.329242 & $0.116^{*}$ & \\
\hline $\mathrm{C} 2$ & $0.8361(3)$ & $0.2860(5)$ & $0.5198(7)$ & $0.1100(17)$ & \\
\hline $\mathrm{H} 2 \mathrm{~A}$ & 0.880352 & 0.293117 & 0.526112 & $0.132 *$ & \\
\hline $\mathrm{C} 3$ & $0.8032(3)$ & $0.2223(5)$ & $0.6272(7)$ & $0.1098(16)$ & \\
\hline $\mathrm{H} 3$ & 0.826349 & 0.187242 & 0.704234 & $0.132 *$ & \\
\hline $\mathrm{C} 4$ & $0.7380(3)$ & $0.2085(4)$ & $0.6256(6)$ & 0.0957 (13) & \\
\hline $\mathrm{H} 4$ & 0.716824 & 0.165147 & 0.698781 & $0.115^{*}$ & \\
\hline $\mathrm{C} 5$ & $0.7054(2)$ & $0.2630(3)$ & $0.5081(4)$ & $0.0724(10)$ & \\
\hline C6 & $0.7375(2)$ & $0.3280(3)$ & $0.3992(5)$ & $0.0748(10)$ & \\
\hline $\mathrm{C} 7$ & $0.6375(2)$ & $0.3395(3)$ & $0.3484(4)$ & $0.0652(10)$ & \\
\hline $\mathrm{C} 8$ & $0.57530(16)$ & $0.3683(3)$ & $0.2821(4)$ & $0.0605(8)$ & \\
\hline C9 & $0.52110(19)$ & $0.3027(3)$ & $0.3118(4)$ & $0.0679(10)$ & \\
\hline H9 & 0.523936 & 0.237162 & 0.373482 & $0.081^{*}$ & \\
\hline $\mathrm{C} 10$ & $0.46302(19)$ & $0.3338(3)$ & 0.2509 (4) & $0.0785(11)$ & \\
\hline H10 & 0.427035 & 0.288323 & 0.270114 & $0.094 *$ & \\
\hline C11 & 0.45811 (19) & $0.4303(4)$ & 0.1630 (4) & $0.0716(10)$ & \\
\hline $\mathrm{C} 12$ & $0.5104(2)$ & $0.4970(4)$ & $0.1317(5)$ & $0.0783(11)$ & \\
\hline H12 & 0.506725 & 0.562819 & 0.070782 & $0.094 *$ & \\
\hline $\mathrm{C} 13$ & $0.5690(2)$ & $0.4659(3)$ & 0.1912 (4) & $0.0752(11)$ & \\
\hline H13 & 0.604801 & 0.511332 & 0.169824 & $0.090^{*}$ & \\
\hline C14 & $0.3486(2)$ & $0.4793(3)$ & $0.1868(5)$ & $0.0734(11)$ & \\
\hline $\mathrm{C} 15$ & $0.2890(2)$ & $0.4466(3)$ & $0.1358(5)$ & 0.0804 (11) & \\
\hline H15 & 0.284932 & 0.408992 & 0.043566 & $0.096^{*}$ & \\
\hline C16 & $0.2358(2)$ & 0.4694 (4) & $0.2210(6)$ & $0.0827(12)$ & \\
\hline
\end{tabular}




\begin{tabular}{|c|c|c|c|c|c|}
\hline $\mathrm{C} 17$ & $0.2414(2)$ & $0.5253(4)$ & $0.3584(5)$ & $0.0845(12)$ & \\
\hline C18 & 0.3018 (2) & $0.5574(4)$ & $0.4094(5)$ & $0.0890(12)$ & \\
\hline H18 & 0.306198 & 0.594294 & 0.502000 & $0.107^{*}$ & \\
\hline C19 & $0.3549(2)$ & 0.5347 (4) & $0.3231(5)$ & $0.0820(11)$ & \\
\hline H19 & 0.395119 & 0.556988 & 0.357067 & $0.098^{*}$ & \\
\hline $\mathrm{C} 20$ & $0.1739(3)$ & 0.4332 (4) & $0.1682(6)$ & $0.1069(17)$ & \\
\hline $\mathrm{C} 21$ & $0.1857(3)$ & $0.5498(5)$ & $0.4466(6)$ & $0.1113(17)$ & \\
\hline $\mathrm{C} 22$ & $0.5253(2)$ & 0.0068 (4) & 0.8099 (6) & $0.1185(17)$ & \\
\hline $\mathrm{H} 22 \mathrm{~A}$ & 0.536012 & -0.018040 & 0.909946 & $0.178^{*}$ & $0.623(5)$ \\
\hline $\mathrm{H} 22 \mathrm{~B}$ & 0.479713 & 0.005567 & 0.797600 & $0.178^{*}$ & $0.623(5)$ \\
\hline $\mathrm{H} 22 \mathrm{C}$ & 0.544633 & -0.045215 & 0.738362 & $0.178^{*}$ & $0.623(5)$ \\
\hline $\mathrm{H} 22 \mathrm{D}$ & 0.497256 & -0.022369 & 0.886737 & $0.178^{*}$ & $0.377(5)$ \\
\hline $\mathrm{H} 22 \mathrm{E}$ & 0.527035 & -0.048198 & 0.728417 & $0.178^{*}$ & $0.377(5)$ \\
\hline $\mathrm{H} 22 \mathrm{~F}$ & 0.567387 & 0.017228 & 0.850914 & $0.178^{*}$ & $0.377(5)$ \\
\hline $\mathrm{C} 23$ & $0.4951(4)$ & $0.2180(5)$ & $0.8988(7)$ & $0.151(2)$ & \\
\hline $\mathrm{H} 23 \mathrm{~A}$ & 0.502326 & 0.196930 & 1.001908 & $0.227^{*}$ & $0.623(5)$ \\
\hline $\mathrm{H} 23 \mathrm{~B}$ & 0.499347 & 0.301270 & 0.887593 & $0.227^{*}$ & $0.623(5)$ \\
\hline $\mathrm{H} 23 \mathrm{C}$ & 0.452845 & 0.194538 & 0.869547 & $0.227 *$ & $0.623(5)$ \\
\hline $\mathrm{H} 23 \mathrm{D}$ & 0.464660 & 0.183633 & 0.966396 & $0.227^{*}$ & $0.377(5)$ \\
\hline $\mathrm{H} 23 \mathrm{E}$ & 0.536893 & 0.215626 & 0.943443 & $0.227^{*}$ & $0.377(5)$ \\
\hline $\mathrm{H} 23 \mathrm{~F}$ & 0.483454 & 0.297886 & 0.879270 & $0.227^{*}$ & $0.377(5)$ \\
\hline
\end{tabular}

Atomic displacement parameters $\left(\AA^{2}\right)$

\begin{tabular}{lllllll}
\hline & $U^{11}$ & $U^{22}$ & $U^{33}$ & $U^{12}$ & $U^{13}$ & $U^{23}$ \\
\hline S1A & $0.0892(19)$ & $0.1040(13)$ & $0.0676(11)$ & $-0.0240(11)$ & $-0.0011(11)$ & $0.0104(10)$ \\
S1B & $0.124(5)$ & $0.131(3)$ & $0.087(3)$ & $0.016(3)$ & $-0.017(2)$ & $0.002(2)$ \\
O1 & $0.081(2)$ & $0.135(2)$ & $0.0614(15)$ & $0.0085(18)$ & $0.0017(16)$ & $0.0154(17)$ \\
O2 & $0.159(4)$ & $0.159(3)$ & $0.109(3)$ & $0.021(3)$ & $0.056(3)$ & $0.056(2)$ \\
N1 & $0.068(2)$ & $0.0793(19)$ & $0.078(2)$ & $-0.0039(17)$ & $0.0160(19)$ & $-0.0036(19)$ \\
N2 & $0.078(2)$ & $0.0676(19)$ & $0.070(2)$ & $-0.0024(17)$ & $0.0048(19)$ & $-0.0014(16)$ \\
N3 & $0.097(3)$ & $0.166(4)$ & $0.165(4)$ & $-0.038(3)$ & $-0.028(3)$ & $0.051(4)$ \\
N4 & $0.102(4)$ & $0.230(6)$ & $0.135(4)$ & $0.061(4)$ & $0.025(3)$ & $0.029(4)$ \\
C1 & $0.080(3)$ & $0.110(3)$ & $0.100(3)$ & $0.005(3)$ & $0.014(3)$ & $-0.028(3)$ \\
C2 & $0.079(3)$ & $0.138(5)$ & $0.113(4)$ & $0.030(3)$ & $-0.003(4)$ & $-0.042(4)$ \\
C3 & $0.111(5)$ & $0.112(4)$ & $0.106(4)$ & $0.052(3)$ & $-0.008(4)$ & $-0.028(3)$ \\
C4 & $0.103(4)$ & $0.083(3)$ & $0.101(3)$ & $0.023(3)$ & $-0.001(3)$ & $-0.010(3)$ \\
C5 & $0.084(3)$ & $0.062(2)$ & $0.072(3)$ & $0.014(2)$ & $0.001(3)$ & $-0.011(2)$ \\
C6 & $0.068(3)$ & $0.078(3)$ & $0.078(3)$ & $0.006(2)$ & $0.009(2)$ & $-0.019(2)$ \\
C7 & $0.079(3)$ & $0.0538(19)$ & $0.063(2)$ & $-0.001(2)$ & $0.011(2)$ & $-0.0006(19)$ \\
C8 & $0.070(2)$ & $0.0558(19)$ & $0.0556(19)$ & $-0.0026(18)$ & $0.010(2)$ & $-0.0016(19)$ \\
C9 & $0.079(3)$ & $0.064(2)$ & $0.061(2)$ & $-0.009(2)$ & $0.008(2)$ & $0.0118(18)$ \\
C10 & $0.075(3)$ & $0.085(3)$ & $0.076(3)$ & $-0.013(2)$ & $0.008(2)$ & $0.010(2)$ \\
C11 & $0.071(3)$ & $0.087(3)$ & $0.057(2)$ & $0.007(2)$ & $0.006(2)$ & $0.010(2)$ \\
C12 & $0.084(3)$ & $0.076(3)$ & $0.075(3)$ & $0.002(2)$ & $0.007(2)$ & $0.020(2)$ \\
C13 & $0.081(3)$ & $0.067(2)$ & $0.077(3)$ & $-0.008(2)$ & $0.011(2)$ & $0.007(2)$ \\
C14 & $0.074(3)$ & $0.085(3)$ & $0.062(2)$ & $0.003(2)$ & $-0.004(2)$ & $0.022(2)$ \\
C15 & $0.084(3)$ & $0.086(3)$ & $0.071(3)$ & $-0.005(2)$ & $-0.013(2)$ & $0.020(2)$ \\
& & & & & &
\end{tabular}




\begin{tabular}{lllllll}
\hline C16 & $0.067(3)$ & $0.087(3)$ & $0.094(3)$ & $-0.004(2)$ & $-0.007(3)$ & $0.037(3)$ \\
$\mathrm{C} 17$ & $0.075(3)$ & $0.099(3)$ & $0.079(3)$ & $0.016(2)$ & $0.000(3)$ & $0.029(3)$ \\
$\mathrm{C} 18$ & $0.088(4)$ & $0.106(3)$ & $0.073(3)$ & $0.021(3)$ & $-0.007(3)$ & $0.007(3)$ \\
$\mathrm{C} 19$ & $0.073(3)$ & $0.103(3)$ & $0.071(3)$ & $0.004(2)$ & $-0.009(2)$ & $0.007(2)$ \\
$\mathrm{C} 20$ & $0.085(3)$ & $0.117(4)$ & $0.119(4)$ & $-0.012(3)$ & $-0.012(3)$ & $0.050(3)$ \\
$\mathrm{C} 21$ & $0.089(4)$ & $0.139(4)$ & $0.106(4)$ & $0.034(3)$ & $0.008(3)$ & $0.034(3)$ \\
$\mathrm{C} 22$ & $0.150(5)$ & $0.096(3)$ & $0.110(4)$ & $-0.009(3)$ & $0.006(3)$ & $0.021(3)$ \\
$\mathrm{C} 23$ & $0.231(7)$ & $0.101(4)$ & $0.122(4)$ & $0.003(4)$ & $0.046(5)$ & $-0.007(4)$ \\
\hline
\end{tabular}

Geometric parameters $\left(\AA,{ }^{\circ}\right)$

\begin{tabular}{|c|c|c|c|}
\hline $\mathrm{S} 1 \mathrm{~A}-\mathrm{O} 2$ & $1.434(4)$ & $\mathrm{C} 10-\mathrm{C} 11$ & $1.356(5)$ \\
\hline $\mathrm{S} 1 \mathrm{~A}-\mathrm{C} 22$ & $1.706(5)$ & $\mathrm{C} 10-\mathrm{H} 10$ & 0.9300 \\
\hline $\mathrm{S} 1 \mathrm{~A}-\mathrm{C} 23$ & $1.800(6)$ & $\mathrm{C} 11-\mathrm{C} 12$ & $1.362(5)$ \\
\hline $\mathrm{S} 1 \mathrm{~B}-\mathrm{O} 2$ & $1.379(5)$ & $\mathrm{C} 12-\mathrm{C} 13$ & $1.381(5)$ \\
\hline $\mathrm{S} 1 \mathrm{~B}-\mathrm{C} 23$ & $1.624(7)$ & $\mathrm{C} 12-\mathrm{H} 12$ & 0.9300 \\
\hline $\mathrm{S} 1 \mathrm{~B}-\mathrm{C} 22$ & $1.801(6)$ & $\mathrm{C} 13-\mathrm{H} 13$ & 0.9300 \\
\hline $\mathrm{O} 1-\mathrm{C} 14$ & $1.367(5)$ & $\mathrm{C} 14-\mathrm{C} 19$ & $1.374(5)$ \\
\hline $\mathrm{O} 1-\mathrm{C} 11$ & $1.406(4)$ & $\mathrm{C} 14-\mathrm{C} 15$ & $1.378(5)$ \\
\hline $\mathrm{N} 1-\mathrm{C} 7$ & $1.314(4)$ & $\mathrm{C} 15-\mathrm{C} 16$ & $1.372(6)$ \\
\hline $\mathrm{N} 1-\mathrm{C} 6$ & $1.388(5)$ & $\mathrm{C} 15-\mathrm{H} 15$ & 0.9300 \\
\hline $\mathrm{N} 2-\mathrm{C} 7$ & $1.361(4)$ & $\mathrm{C} 16-\mathrm{C} 17$ & $1.384(6)$ \\
\hline $\mathrm{N} 2-\mathrm{C} 5$ & $1.365(5)$ & $\mathrm{C} 16-\mathrm{C} 20$ & $1.437(7)$ \\
\hline $\mathrm{N} 2-\mathrm{H} 2$ & 0.8600 & $\mathrm{C} 17-\mathrm{C} 18$ & $1.390(6)$ \\
\hline $\mathrm{N} 3-\mathrm{C} 20$ & $1.137(6)$ & $\mathrm{C} 17-\mathrm{C} 21$ & $1.433(7)$ \\
\hline $\mathrm{N} 4-\mathrm{C} 21$ & $1.132(6)$ & $\mathrm{C} 18-\mathrm{C} 19$ & $1.375(5)$ \\
\hline $\mathrm{C} 1-\mathrm{C} 6$ & $1.384(6)$ & C18-H18 & 0.9300 \\
\hline $\mathrm{C} 1-\mathrm{C} 2$ & $1.384(7)$ & C19-H19 & 0.9300 \\
\hline $\mathrm{C} 1-\mathrm{H} 1$ & 0.9300 & $\mathrm{C} 22-\mathrm{H} 22 \mathrm{~A}$ & 0.9600 \\
\hline $\mathrm{C} 2-\mathrm{C} 3$ & $1.384(7)$ & $\mathrm{C} 22-\mathrm{H} 22 \mathrm{~B}$ & 0.9600 \\
\hline $\mathrm{C} 2-\mathrm{H} 2 \mathrm{~A}$ & 0.9300 & $\mathrm{C} 22-\mathrm{H} 22 \mathrm{C}$ & 0.9600 \\
\hline $\mathrm{C} 3-\mathrm{C} 4$ & $1.372(7)$ & $\mathrm{C} 22-\mathrm{H} 22 \mathrm{D}$ & 0.9600 \\
\hline $\mathrm{C} 3-\mathrm{H} 3$ & 0.9300 & $\mathrm{C} 22-\mathrm{H} 22 \mathrm{E}$ & 0.9600 \\
\hline $\mathrm{C} 4-\mathrm{C} 5$ & $1.395(6)$ & $\mathrm{C} 22-\mathrm{H} 22 \mathrm{~F}$ & 0.9600 \\
\hline $\mathrm{C} 4-\mathrm{H} 4$ & 0.9300 & $\mathrm{C} 23-\mathrm{H} 23 \mathrm{~A}$ & 0.9600 \\
\hline $\mathrm{C} 5-\mathrm{C} 6$ & $1.392(5)$ & $\mathrm{C} 23-\mathrm{H} 23 \mathrm{~B}$ & 0.9600 \\
\hline $\mathrm{C} 7-\mathrm{C} 8$ & $1.466(5)$ & $\mathrm{C} 23-\mathrm{H} 23 \mathrm{C}$ & 0.9600 \\
\hline $\mathrm{C} 8-\mathrm{C} 9$ & $1.384(5)$ & $\mathrm{C} 23-\mathrm{H} 23 \mathrm{D}$ & 0.9600 \\
\hline $\mathrm{C} 8-\mathrm{C} 13$ & $1.384(5)$ & $\mathrm{C} 23-\mathrm{H} 23 \mathrm{E}$ & 0.9600 \\
\hline $\mathrm{C} 9-\mathrm{C} 10$ & $1.376(5)$ & $\mathrm{C} 23-\mathrm{H} 23 \mathrm{~F}$ & 0.9600 \\
\hline C9-H9 & 0.9300 & & \\
\hline $\mathrm{O} 2-\mathrm{S} 1 \mathrm{~A}-\mathrm{C} 22$ & $110.0(3)$ & $\mathrm{C} 12-\mathrm{C} 13-\mathrm{C} 8$ & $121.0(4)$ \\
\hline $\mathrm{O} 2-\mathrm{S} 1 \mathrm{~A}-\mathrm{C} 23$ & $104.1(3)$ & $\mathrm{C} 12-\mathrm{C} 13-\mathrm{H} 13$ & 119.5 \\
\hline $\mathrm{C} 22-\mathrm{S} 1 \mathrm{~A}-\mathrm{C} 23$ & $96.5(3)$ & $\mathrm{C} 8-\mathrm{C} 13-\mathrm{H} 13$ & 119.5 \\
\hline $\mathrm{O} 2-\mathrm{S} 1 \mathrm{~B}-\mathrm{C} 23$ & $116.7(4)$ & $\mathrm{O} 1-\mathrm{C} 14-\mathrm{C} 19$ & $122.5(4)$ \\
\hline $\mathrm{O} 2-\mathrm{S} 1 \mathrm{~B}-\mathrm{C} 22$ & $107.6(4)$ & $\mathrm{O} 1-\mathrm{C} 14-\mathrm{C} 15$ & $117.4(4)$ \\
\hline $\mathrm{C} 23-\mathrm{S} 1 \mathrm{~B}-\mathrm{C} 22$ & $99.4(3)$ & $\mathrm{C} 19-\mathrm{C} 14-\mathrm{C} 15$ & $120.1(4)$ \\
\hline
\end{tabular}




\begin{tabular}{|c|c|c|c|}
\hline $\mathrm{C} 14-\mathrm{O} 1-\mathrm{C} 11$ & $117.2(3)$ & $\mathrm{C} 16-\mathrm{C} 15-\mathrm{C} 14$ & $120.1(4)$ \\
\hline $\mathrm{C} 7-\mathrm{N} 1-\mathrm{C} 6$ & $104.9(3)$ & $\mathrm{C} 16-\mathrm{C} 15-\mathrm{H} 15$ & 120.0 \\
\hline $\mathrm{C} 7-\mathrm{N} 2-\mathrm{C} 5$ & $106.9(3)$ & $\mathrm{C} 14-\mathrm{C} 15-\mathrm{H} 15$ & 120.0 \\
\hline $\mathrm{C} 7-\mathrm{N} 2-\mathrm{H} 2$ & 126.5 & $\mathrm{C} 15-\mathrm{C} 16-\mathrm{C} 17$ & $120.3(4)$ \\
\hline $\mathrm{C} 5-\mathrm{N} 2-\mathrm{H} 2$ & 126.5 & $\mathrm{C} 15-\mathrm{C} 16-\mathrm{C} 20$ & $119.8(5)$ \\
\hline $\mathrm{C} 6-\mathrm{C} 1-\mathrm{C} 2$ & $118.1(5)$ & $\mathrm{C} 17-\mathrm{C} 16-\mathrm{C} 20$ & $119.9(5)$ \\
\hline $\mathrm{C} 6-\mathrm{C} 1-\mathrm{H} 1$ & 120.9 & $\mathrm{C} 16-\mathrm{C} 17-\mathrm{C} 18$ & $119.2(5)$ \\
\hline $\mathrm{C} 2-\mathrm{C} 1-\mathrm{H} 1$ & 120.9 & $\mathrm{C} 16-\mathrm{C} 17-\mathrm{C} 21$ & $120.3(5)$ \\
\hline $\mathrm{C} 1-\mathrm{C} 2-\mathrm{C} 3$ & $120.1(5)$ & $\mathrm{C} 18-\mathrm{C} 17-\mathrm{C} 21$ & $120.6(5)$ \\
\hline $\mathrm{C} 1-\mathrm{C} 2-\mathrm{H} 2 \mathrm{~A}$ & 120.0 & $\mathrm{C} 19-\mathrm{C} 18-\mathrm{C} 17$ & $120.1(4)$ \\
\hline $\mathrm{C} 3-\mathrm{C} 2-\mathrm{H} 2 \mathrm{~A}$ & 120.0 & $\mathrm{C} 19-\mathrm{C} 18-\mathrm{H} 18$ & 119.9 \\
\hline $\mathrm{C} 4-\mathrm{C} 3-\mathrm{C} 2$ & $123.3(5)$ & $\mathrm{C} 17-\mathrm{C} 18-\mathrm{H} 18$ & 119.9 \\
\hline $\mathrm{C} 4-\mathrm{C} 3-\mathrm{H} 3$ & 118.4 & $\mathrm{C} 14-\mathrm{C} 19-\mathrm{C} 18$ & $120.2(4)$ \\
\hline $\mathrm{C} 2-\mathrm{C} 3-\mathrm{H} 3$ & 118.4 & $\mathrm{C} 14-\mathrm{C} 19-\mathrm{H} 19$ & 119.9 \\
\hline $\mathrm{C} 3-\mathrm{C} 4-\mathrm{C} 5$ & $116.2(5)$ & $\mathrm{C} 18-\mathrm{C} 19-\mathrm{H} 19$ & 119.9 \\
\hline $\mathrm{C} 3-\mathrm{C} 4-\mathrm{H} 4$ & 121.9 & $\mathrm{~N} 3-\mathrm{C} 20-\mathrm{C} 16$ & $179.4(6)$ \\
\hline $\mathrm{C} 5-\mathrm{C} 4-\mathrm{H} 4$ & 121.9 & $\mathrm{~N} 4-\mathrm{C} 21-\mathrm{C} 17$ & $177.9(7)$ \\
\hline $\mathrm{N} 2-\mathrm{C} 5-\mathrm{C} 6$ & $105.9(4)$ & $\mathrm{S} 1 \mathrm{~A}-\mathrm{C} 22-\mathrm{H} 22 \mathrm{~A}$ & 109.5 \\
\hline $\mathrm{N} 2-\mathrm{C} 5-\mathrm{C} 4$ & $132.5(4)$ & $\mathrm{S} 1 \mathrm{~A}-\mathrm{C} 22-\mathrm{H} 22 \mathrm{~B}$ & 109.5 \\
\hline $\mathrm{C} 6-\mathrm{C} 5-\mathrm{C} 4$ & $121.6(5)$ & $\mathrm{H} 22 \mathrm{~A}-\mathrm{C} 22-\mathrm{H} 22 \mathrm{~B}$ & 109.5 \\
\hline $\mathrm{C} 1-\mathrm{C} 6-\mathrm{N} 1$ & $129.8(4)$ & $\mathrm{S} 1 \mathrm{~A}-\mathrm{C} 22-\mathrm{H} 22 \mathrm{C}$ & 109.5 \\
\hline $\mathrm{C} 1-\mathrm{C} 6-\mathrm{C} 5$ & $120.7(4)$ & $\mathrm{H} 22 \mathrm{~A}-\mathrm{C} 22-\mathrm{H} 22 \mathrm{C}$ & 109.5 \\
\hline $\mathrm{N} 1-\mathrm{C} 6-\mathrm{C} 5$ & $109.4(4)$ & $\mathrm{H} 22 \mathrm{~B}-\mathrm{C} 22-\mathrm{H} 22 \mathrm{C}$ & 109.5 \\
\hline $\mathrm{N} 1-\mathrm{C} 7-\mathrm{N} 2$ & $112.8(4)$ & $\mathrm{S} 1 \mathrm{~B}-\mathrm{C} 22-\mathrm{H} 22 \mathrm{D}$ & 109.5 \\
\hline $\mathrm{N} 1-\mathrm{C} 7-\mathrm{C} 8$ & $126.0(3)$ & $\mathrm{S} 1 \mathrm{~B}-\mathrm{C} 22-\mathrm{H} 22 \mathrm{E}$ & 109.5 \\
\hline $\mathrm{N} 2-\mathrm{C} 7-\mathrm{C} 8$ & $121.2(3)$ & $\mathrm{H} 22 \mathrm{D}-\mathrm{C} 22-\mathrm{H} 22 \mathrm{E}$ & 109.5 \\
\hline $\mathrm{C} 9-\mathrm{C} 8-\mathrm{C} 13$ & $118.0(4)$ & $\mathrm{S} 1 \mathrm{~B}-\mathrm{C} 22-\mathrm{H} 22 \mathrm{~F}$ & 109.5 \\
\hline $\mathrm{C} 9-\mathrm{C} 8-\mathrm{C} 7$ & $122.0(3)$ & $\mathrm{H} 22 \mathrm{D}-\mathrm{C} 22-\mathrm{H} 22 \mathrm{~F}$ & 109.5 \\
\hline $\mathrm{C} 13-\mathrm{C} 8-\mathrm{C} 7$ & $120.0(3)$ & $\mathrm{H} 22 \mathrm{E}-\mathrm{C} 22-\mathrm{H} 22 \mathrm{~F}$ & 109.5 \\
\hline $\mathrm{C} 10-\mathrm{C} 9-\mathrm{C} 8$ & $120.6(4)$ & $\mathrm{S} 1 \mathrm{~A}-\mathrm{C} 23-\mathrm{H} 23 \mathrm{~A}$ & 109.5 \\
\hline $\mathrm{C} 10-\mathrm{C} 9-\mathrm{H} 9$ & 119.7 & $\mathrm{~S} 1 \mathrm{~A}-\mathrm{C} 23-\mathrm{H} 23 \mathrm{~B}$ & 109.5 \\
\hline $\mathrm{C} 8-\mathrm{C} 9-\mathrm{H} 9$ & 119.7 & $\mathrm{H} 23 \mathrm{~A}-\mathrm{C} 23-\mathrm{H} 23 \mathrm{~B}$ & 109.5 \\
\hline $\mathrm{C} 11-\mathrm{C} 10-\mathrm{C} 9$ & $120.2(4)$ & $\mathrm{S} 1 \mathrm{~A}-\mathrm{C} 23-\mathrm{H} 23 \mathrm{C}$ & 109.5 \\
\hline $\mathrm{C} 11-\mathrm{C} 10-\mathrm{H} 10$ & 119.9 & $\mathrm{H} 23 \mathrm{~A}-\mathrm{C} 23-\mathrm{H} 23 \mathrm{C}$ & 109.5 \\
\hline $\mathrm{C} 9-\mathrm{C} 10-\mathrm{H} 10$ & 119.9 & $\mathrm{H} 23 \mathrm{~B}-\mathrm{C} 23-\mathrm{H} 23 \mathrm{C}$ & 109.5 \\
\hline $\mathrm{C} 10-\mathrm{C} 11-\mathrm{C} 12$ & $120.8(4)$ & $\mathrm{S} 1 \mathrm{~B}-\mathrm{C} 23-\mathrm{H} 23 \mathrm{D}$ & 109.5 \\
\hline $\mathrm{C} 10-\mathrm{C} 11-\mathrm{O} 1$ & $120.3(4)$ & $\mathrm{S} 1 \mathrm{~B}-\mathrm{C} 23-\mathrm{H} 23 \mathrm{E}$ & 109.5 \\
\hline $\mathrm{C} 12-\mathrm{C} 11-\mathrm{O} 1$ & $118.8(4)$ & $\mathrm{H} 23 \mathrm{D}-\mathrm{C} 23-\mathrm{H} 23 \mathrm{E}$ & 109.5 \\
\hline $\mathrm{C} 11-\mathrm{C} 12-\mathrm{C} 13$ & $119.4(4)$ & $\mathrm{S} 1 \mathrm{~B}-\mathrm{C} 23-\mathrm{H} 23 \mathrm{~F}$ & 109.5 \\
\hline $\mathrm{C} 11-\mathrm{C} 12-\mathrm{H} 12$ & 120.3 & $\mathrm{H} 23 \mathrm{D}-\mathrm{C} 23-\mathrm{H} 23 \mathrm{~F}$ & 109.5 \\
\hline $\mathrm{C} 13-\mathrm{C} 12-\mathrm{H} 12$ & 120.3 & $\mathrm{H} 23 \mathrm{E}-\mathrm{C} 23-\mathrm{H} 23 \mathrm{~F}$ & 109.5 \\
\hline $\mathrm{C} 6-\mathrm{C} 1-\mathrm{C} 2-\mathrm{C} 3$ & $1.1(7)$ & $\mathrm{C} 8-\mathrm{C} 9-\mathrm{C} 10-\mathrm{C} 11$ & $-1.2(6)$ \\
\hline $\mathrm{C} 1-\mathrm{C} 2-\mathrm{C} 3-\mathrm{C} 4$ & $-0.3(7)$ & $\mathrm{C} 9-\mathrm{C} 10-\mathrm{C} 11-\mathrm{C} 12$ & $1.0(6)$ \\
\hline $\mathrm{C} 2-\mathrm{C} 3-\mathrm{C} 4-\mathrm{C} 5$ & $-0.2(7)$ & $\mathrm{C} 9-\mathrm{C} 10-\mathrm{C} 11-\mathrm{O} 1$ & $176.6(3)$ \\
\hline $\mathrm{C} 7-\mathrm{N} 2-\mathrm{C} 5-\mathrm{C} 6$ & $-1.6(4)$ & $\mathrm{C} 14-\mathrm{O} 1-\mathrm{C} 11-\mathrm{C} 10$ & $60.5(5)$ \\
\hline $\mathrm{C} 7-\mathrm{N} 2-\mathrm{C} 5-\mathrm{C} 4$ & $177.2(4)$ & $\mathrm{C} 14-\mathrm{O} 1-\mathrm{C} 11-\mathrm{C} 12$ & $-123.7(4)$ \\
\hline $\mathrm{C} 3-\mathrm{C} 4-\mathrm{C} 5-\mathrm{N} 2$ & $-178.8(4)$ & $\mathrm{C} 10-\mathrm{C} 11-\mathrm{C} 12-\mathrm{C} 13$ & $-0.4(6)$ \\
\hline
\end{tabular}




$\begin{array}{llll}\mathrm{C} 3-\mathrm{C} 4-\mathrm{C} 5-\mathrm{C} 6 & -0.1(6) & \mathrm{O} 1-\mathrm{C} 11-\mathrm{C} 12-\mathrm{C} 13 & -176.1(4) \\ \mathrm{C} 2-\mathrm{C} 1-\mathrm{C} 6-\mathrm{N} 1 & 177.1(4) & \mathrm{C} 11-\mathrm{C} 12-\mathrm{C} 13-\mathrm{C} 8 & -0.1(6) \\ \mathrm{C} 2-\mathrm{C} 1-\mathrm{C} 6-\mathrm{C} 5 & -1.4(6) & \mathrm{C} 9-\mathrm{C} 8-\mathrm{C} 13-\mathrm{C} 12 & -0.1(5) \\ \mathrm{C} 7-\mathrm{N} 1-\mathrm{C} 6-\mathrm{C} 1 & -178.8(4) & \mathrm{C} 7-\mathrm{C} 8-\mathrm{C} 13-\mathrm{C} 12 & -177.8(4) \\ \mathrm{C} 7-\mathrm{N} 1-\mathrm{C} 6-\mathrm{C} 5 & -0.2(4) & \mathrm{C} 11-\mathrm{O} 1-\mathrm{C} 14-\mathrm{C} 19 & 36.2(5) \\ \mathrm{N} 2-\mathrm{C} 5-\mathrm{C} 6-\mathrm{C} 1 & 179.9(3) & \mathrm{C} 11-\mathrm{O} 1-\mathrm{C} 14-\mathrm{C} 15 & -146.4(3) \\ \mathrm{C} 4-\mathrm{C} 5-\mathrm{C} 6-\mathrm{C} 1 & 0.9(6) & \mathrm{O} 1-\mathrm{C} 14-\mathrm{C} 15-\mathrm{C} 16 & -177.3(3) \\ \mathrm{N} 2-\mathrm{C} 5-\mathrm{C} 6-\mathrm{N} 1 & 1.1(4) & \mathrm{C} 19-\mathrm{C} 14-\mathrm{C} 15-\mathrm{C} 16 & 0.1(6) \\ \mathrm{C} 4-\mathrm{C} 5-\mathrm{C} 6-\mathrm{N} 1 & -177.9(3) & \mathrm{C} 14-\mathrm{C} 15-\mathrm{C} 16-\mathrm{C} 17 & 0.0(6) \\ \mathrm{C} 6-\mathrm{N} 1-\mathrm{C} 7-\mathrm{N} 2 & -0.9(4) & \mathrm{C} 14-\mathrm{C} 15-\mathrm{C} 16-\mathrm{C} 20 & -178.9(4) \\ \mathrm{C} 6-\mathrm{N} 1-\mathrm{C} 7-\mathrm{C} 8 & 178.9(3) & \mathrm{C} 15-\mathrm{C} 16-\mathrm{C} 17-\mathrm{C} 18 & -0.3(6) \\ \mathrm{C} 5-\mathrm{N} 2-\mathrm{C} 7-\mathrm{N} 1 & 1.6(4) & \mathrm{C} 20-\mathrm{C} 16-\mathrm{C} 17-\mathrm{C} 18 & 178.6(4) \\ \mathrm{C} 5-\mathrm{N} 2-\mathrm{C} 7-\mathrm{C} 8 & -178.1(3) & \mathrm{C} 15-\mathrm{C} 16-\mathrm{C} 17-\mathrm{C} 21 & 179.5(4) \\ \mathrm{N} 1-\mathrm{C} 7-\mathrm{C} 8-\mathrm{C} 9 & 161.5(3) & \mathrm{C} 20-\mathrm{C} 16-\mathrm{C} 17-\mathrm{C} 21 & -1.6(6) \\ \mathrm{N} 2-\mathrm{C} 7-\mathrm{C} 8-\mathrm{C} 9 & -18.8(5) & \mathrm{C} 16-\mathrm{C} 17-\mathrm{C} 18-\mathrm{C} 19 & 0.6(6) \\ \mathrm{N} 1-\mathrm{C} 7-\mathrm{C} 8-\mathrm{C} 13 & -20.9(5) & \mathrm{C} 21-\mathrm{C} 17-\mathrm{C} 18-\mathrm{C} 19 & -179.2(4) \\ \mathrm{N} 2-\mathrm{C} 7-\mathrm{C} 8-\mathrm{C} 13 & 158.9(3) & \mathrm{O} 1-\mathrm{C} 14-\mathrm{C} 19-\mathrm{C} 18 & 177.5(4) \\ \mathrm{C} 13-\mathrm{C} 8-\mathrm{C} 9-\mathrm{C} 10 & 0.7(5) & \mathrm{C} 15-\mathrm{C} 14-\mathrm{C} 19-\mathrm{C} 18 & 0.2(5) \\ \mathrm{C} 7-\mathrm{C} 8-\mathrm{C} 9-\mathrm{C} 10 & 178.3(3) & \mathrm{C} 17-\mathrm{C} 18-\mathrm{C} 19-\mathrm{C} 14 & -0.6(6)\end{array}$

Hydrogen-bond geometry $\left(\AA,{ }^{\circ}\right)$

\begin{tabular}{lllll}
\hline$D-\mathrm{H} \cdots A$ & $D-\mathrm{H}$ & $\mathrm{H} \cdots A$ & $D \cdots A$ & $D-\mathrm{H} \cdots A$ \\
\hline $\mathrm{N} 2-\mathrm{H} 2 \cdots \mathrm{O} 2$ & 0.86 & 1.94 & $2.794(5)$ & 172 \\
$\mathrm{~N} 2-\mathrm{H} 2 \cdots \mathrm{S} 1 A$ & 0.86 & 2.83 & $3.614(4)$ & 152 \\
$\mathrm{C} 9-\mathrm{H} 9 \cdots \mathrm{O} 2$ & 0.93 & 2.40 & $3.175(5)$ & 141 \\
$\mathrm{C} 23-\mathrm{H} 23 D \cdots \mathrm{N} 4{ }^{\mathrm{i}}$ & 0.96 & 2.63 & $3.500(9)$ & 151 \\
\hline
\end{tabular}

Symmetry code: (i) $-x+1 / 2, y-1 / 2, z+1 / 2$. 